\title{
Overexpression of PDK4 is associated with cell proliferation, drug resistance and poor prognosis in ovarian cancer
}

This article was published in the following Dove Press journal: Cancer Management and Research

\author{
Jinghao Wang' \\ Yu Qian² \\ Meiyan $\mathrm{Gao}^{3}$ \\ 'Department of Gynecology and \\ Obstetrics, The Second Hospital, \\ Shanxi Medical University, Taiyuan, \\ Shanxi 03000I, China; ${ }^{2}$ Translational \\ Medicine Research Center, Shanxi \\ Medical University, Taiyuan, Shanxi \\ 03000 I, China; ${ }^{3}$ Department of \\ Biotherapy, Shanxi Provincial Cancer \\ Hospital, Taiyuan, Shanxi 030013 \\ China
}

Correspondence: Meiyan Gao Department of Biotherapy, Shanxi Provincial Cancer Hospital, No. 3, Staff New Village, Xinghualing District, Taiyuan, Shanxi 030013, China

$\mathrm{Tel}+86035$ I488 I6II

Email ymgaosx@163.com
Purpose: Ovarian cancer is a major type of gynecological malignancy which characterized by the chemoresistance, heterogeneity and highly metastasis. However, the mechanism underlying the progression of ovarian cancer remains elusive. Pyruvate dehydrogenase kinase family plays critical roles in tumorigenesis, and PDK4 has been demonstrated to be an oncogene in many types of cancers. The aim of this study was to identify the role of PDK4 in ovarian cancer.

Methods: We explored the PDK4 expression according to the public database containing patients with different effect of chemotherapy. Cell proliferation and invasion assays were used to determine the function of PDK4. Mice xenograft experiment was conducted to test the protumorigenesis function of PDK4 in vivo. Cell apoptosis under treatment of chemo drugs was detected by flow cytometry and TUNEL analysis. Spheroid formation assay and CD133+ cell population were used to determine the PDK4-induced stem-like traits. Immunohistochemical staining was performed to test the expression of PDK4 in ovarian cancer tissues, and KaplanMeier curve with log-rank test was performed to determine the association between PDK4 expression and ovarian cancer patients' prognosis.

Results: Overexpression of PDK4 markedly promoted cell proliferation, invasion and tumor growth in vivo. Furthermore, PDK4 confers cell resistant to chemotherapy-induced apoptosis. Mechanically, we demonstrated that PDK4 induced stem-like traits. Meanwhile, PDK4 expression was significantly evaluated in ovarian cancer tissues compared to that in adjacent non-cancer tissues, and high expression of PDK4 was associated with poor overall survival and progression-free survival of ovarian cancer patients.

Conclusion: These results identify a novel role of PDK4 in regulating cell stem-like trait, which directly enhances the cell proliferation, invasion and chemoresistance in ovarian cancer, and targeting PDK4 could be a potential approach for ovarian cancer treatments.

Keywords: PDK4, chemoresistance, prognosis, ovarian cancer, stemness

\section{Introduction}

Ovarian cancer is one of the most lethal gynecological malignancies with increased morbidity and mortality. ${ }^{1}$ There are $>20,000$ new cases annually, and the 5-year survival rate remains at $45 \% .{ }^{2,3}$ However, most of the patients are diagnosed in advanced stages, which lead to the unfavorable prognosis. The clinical treatments for advanced ovarian cancer are cytoreduction and chemotherapy. ${ }^{4}$ However, $>30 \%$ of ovarian cancer patients have intrinsic resistance to platinum or other chemodrugs, leading to the frequent relapse and metastasis. ${ }^{5}$ The lack of early diagnostic biomarkers and the development of chemoresistance remain great challenge for ovarian cancer. Hence, it is urgently needed to 
improve the understandings in the underlying mechanisms that promote ovarian cancer cell chemoresistance and malignance.

Tumor cells preferred glycolysis even there are enough oxygen, and this metabolism reprogramming is identified as Warburg effect. ${ }^{6}$ Pyruvate dehydrogenase kinases (PDKs) are the enzymes which are responsible for phosphorylating pyruvate dehydrogenase and the metabolic switch from mitochondrial respiration to cytoplasmic glycolysis. ${ }^{7,8}$ Recently, it was considered that this switch played pivotal role in increased drug resistance. PDK4 is located on chromosome 7q21.3, with 11 exons, coding a protein of 411 amino acids. PDK4 plays critical roles in several types of cancers. ${ }^{9-11}$ Srivastava et al reported that PDK4 prevented ROS-mediated retinoblastoma protein $(\mathrm{Rb})$ hypophosphorylation and antitumorigenic actions of PPAR $\gamma .{ }^{12}$ Song et al reported that PDK4 and miR-122 could represent novel therapeutic approach for hepatocellular cancer. ${ }^{13}$ Furthermore, PDK4 could interact with p100/p49, which contains a death domain, to regulate insulin resistance and inhibit cell apoptosis. ${ }^{14}$ Also, PDK4 was reported to bind to the CREB and consequently, trans-activating the expression of Ras homolog enriched in brain (RHEB) and inducing mTORC1 activity. ${ }^{15}$

In the present study, we examined the expression profiles of PDK family members and found that PDK4 was associated with ovarian cancer chemoresistance. Further investigations indicated that overexpression of PDK4 promoted cell proliferation, invasion and resistant paclitaxel- and cisplatininduced apoptosis, while knocking down of PDK4 suppressed cell proliferation and invasion ability. We also demonstrated that PDK4 induced cell stemness properties and CD133+ subpopulation. The tumor formation assay indicated that PDK4 promoted tumorigenesis in vivo. Finally, PDK4 was detected to be upregulated in ovarian cancer tissues and correlated with poor prognosis. Our study provided evidences that PDK4 could be new target for ovarian cancer treatments.

\section{Materials and methods}

\section{Ethics statement and clinical samples}

The study was approved by the Ethical Committee of Shanxi Medical University. Human ovarian cancer tissue microassay containing 100 cases of ovarian cancer tissues and paired adjacent non-cancer tissues was purchased from Xi'an Alenabio Inc. (Xi'an, China).

\section{Cell lines}

The human ovarian cancer cell lines COV504, OVISE, SKOV3, OV90, ES2 and OVCAR4 were obtained from American Type Culture Collection (ATCC, Manassas, VA, USA). Cells were maintained in RPMI-1640 medium (Gibco, Grand Island, NY, USA) supplemented with $10 \% \mathrm{FBS}, 100 \mathrm{U} / \mathrm{mL}$ penicillin and $100 \mu \mathrm{g} / \mathrm{mL}$ streptomycin at $37^{\circ} \mathrm{C}$ in humidified air with $5 \% \mathrm{CO}_{2}$.

\section{Immunohistochemical (IHC) staining}

IHC staining was performed as described previously. ${ }^{16}$ The slides were de-paraffinized in xylene and rehydrated in gradient ethanol. The slides were incubated with 3\% hydrogen peroxide for 10 minutes, followed by antigen retrieving using citric acid buffer for 30 minutes. After blocking with $5 \%$ BSA, the slides were incubated with primary antibody overnight at $4{ }^{\circ} \mathrm{C}$. The slides were washed with PBS and incubated with peroxidase-conjugated secondary antibody for 30 minutes. The IHC staining was visualized using diaminobenzidine reaction, counterstained with hematoxylin, and the images were captured by Zeiss Axioscop light microscope.

\section{Plasmid and siRNAs' transfection}

PDK4 overexpression plasmid pCDNA3.1-PDK4 was obtained from Hanbio Inc. (Shanghai, China). siRNAs were obtained from Genepharm (Shanghai, China), and the sequences targeted were as follows: 5'-GGACGTAAGAGATTCTCAT-3' and 5'-GGATTTGGTGGAGTTCCAT-3' as described previously for targeting PDK $4 .{ }^{15}$ Briefly, $4 \times 10^{5}$ cells were seeded into six-well plate and cultured for 24 hours. The cell transfection was performed using Lipofectamine 2000 (Invitrogen, Carlsbad, CA, USA) according to the manufacturer's protocol. For overexpressing PDK4, $4 \mu \mathrm{g}$ of plasmid was transfected into cells and cultured for 48 hours. Then, $800 \mu \mathrm{g} / \mathrm{mL}$ of G418 (Sigma, St. Louis, MO, USA) was added to select the stably expressed cells. For knocking down of PDK4, 200 pmol of siRNAs was used per well. The cellular detection was performed after 48-hour transfection.

\section{Quantitative real-time PCR (qRT-PCR)}

Total RNA was extracted from cells using Trizol reagent (Invitrogen) according to the manufacturer's recommendation. Two hundred nanograms of mRNA were used, and cDNA was synthesized using PrimeScript RT Master Kit (Takara, Dalian, China). The qRT-PCR was performed on 7500 Real-Time PCR System (Applied Biosystems, Foster City, CA, USA) with SYBR Green methods as described previously. ${ }^{17}$ The primers used are shown in Table S1. GAPDH was used as internal control.

\section{Western blot}

Cells were lysed using RIPA buffer containing protease inhibitor and phosphatase inhibitor cocktail. The total protein con- 
centration was measured by BCA methods. Sixty micrograms of protein were loaded on to and separated by $10 \%$ SDS-PAGE and transferred to PVDF membranes. The membranes were incubated with primary antibodies and then secondary antibody and visualized by ECL methods. The primary antibodies used were as follows: anti-PDK4 (12949-1-AP; ProteinTech, Rosemont, IL, USA) and anti-cleaved caspase 3 (\#9664; Cell Signaling Technology, Danvers, MA, USA). Anti-tubulin (10068-1-AP; ProteinTech) was used as normalized control.

\section{Cell viability assay}

Cells were plated into 96-well plates in a concentration of 2,000 cells/well. For detecting the cell proliferation, MTT method was performed at the indicated time points. Briefly, $20 \mu \mathrm{L}$ of MTT solution was added to each well, and the plate was incubated again for 3 hours, followed by adding DMSO. The absorbance value was measured by spectrophotometer at OD490. For detecting the chemoresistance ability, paclitaxel (range from 0.625 to $20 \mathrm{nM})^{18}$ and cisplatin (range from 0.5 to $16 \mu \mathrm{M})^{19}$ in gradient concentration were used and after 48 hours, the cell viability was measured.

\section{Apoptosis assay}

For cell apoptosis assay, $5 \times 10^{4}$ cells transfected with PDK4 or knocked down PDK4 were treated with paclitaxel (4 nM) and cisplatin $(3 \mu \mathrm{M})$ for 24 hours, respectively. Then, cells were harvested, washed with PBS and stained with Annexin V Apoptosis Detection Kit (BD Biosciences, San Jose, CA, USA) according to the manufacturer's instructions. The percentage of Annexin V-positive cells was analyzed by flow cytometry as described previously. ${ }^{20}$

\section{Invasion assay}

Invasion assay was performed using Transwell chamber embedded with Matrigel (1:5 dilution; BD Bioscience). Indicated cells $\left(5 \times 10^{4}\right)$ were suspended in serum-free medium and added into the upper chambers. The lower chambers were containing medium with $20 \%$ FBS. After culturing for 48 hours, invading cells were fixed, stained with crystal violet, imaged and counted under the microscope.

\section{Immunofluorescence staining}

The control cells and PDK4-overexpressing cells were treated with DMSO and paclitaxel, respectively, for 24 hours. Then, $3 \times 10^{4}$ cells were plated on the cover slide in 24 -well plate and incubated overnight. After paclitaxel treatment, cells were fixed and permeabilized the membrane by Triton X-100 and incubated with anti-cleaved caspase 3 antibody at $4{ }^{\circ} \mathrm{C}$ overnight. Then, the slides were washed and incubated with Alex Fluor 594-conjugated secondary antibody, followed by staining with DAPI. Immunofluorescence was captured and measured as described previously. ${ }^{16}$

\section{Spheroid formation assay}

For culturing the cell spheroid, 5,000 indicated cells (control cells and PDK4 overexpression/knockdown cells) were seeded into the 6-Well Ultra-Low Adherent Plates maintained in DMEM/F12 medium, supplemented with $10 \mu \mathrm{g} / \mathrm{mL}$ bFGF, $20 \mu \mathrm{g} / \mathrm{mL}$ EGF and $1 \times$ B27. After culturing 10 days, the spheroid diameter and number were measured.

\section{Fluorescence-activated cell sorting (FACS) analysis}

Indicated cells $\left(5 \times 10^{4}\right)$ were harvested, washed twice with PBS and incubated with antibody against CD133 (PE-conjugated; eBioscience, San Diego, CA, USA), and the corresponding isotype controls at $4^{\circ} \mathrm{C}$ in the dark for 40 minutes. Subsequently, cells were washed and processed using flow cytometry. The results were analyzed using MoFlo XDP (Beckman Coulter, Fullerton, CA, USA) as described previously. ${ }^{20}$

\section{Xenograft experiment}

The mice experiments were followed the Guidelines of Animal Care of Shanxi Medical University, and the study was approved by the Animal Care Committee of Shanxi Medical University. Indicated cells $\left(1 \times 10^{6}\right)$ were subcutaneously injected into the left oxter of nude mice at 6-week ages. Tumor size was monitored every 3 days. After 4 weeks, the mice were sacrificed and the tumors were removed and paraformaldehyde fixed and paraffin-embedded, then used for IHC to detect the expression of Ki67.

\section{Data source}

The microarray data concerning to the expression of PDKs in 12 platinum-resistant patients and 16 platinum-sensitive patients were acquired from GEO database (GSE51373, https:// www.ncbi.nlm.nih.gov/geo/query/acc.cgi?acc=GSE51373). The clustering algorithm was measured by Cluster 3.0 and Java TreeView. ${ }^{21,22}$ The prognostic value of PDK4 was identified by Kaplan-Meier plot (http://kmplot.com/analysis/index. php p $=$ service \& cancer $=$ ovar) $).{ }^{23}$ The Affy ID was $225207 \_$at, and we chose the item "Auto select best cutoff".

\section{Statistical analysis}

Statistical analysis was performed using SPSS 21.0 (IBM, Chicago, IL, USA), and graphic data were performed using 
GraphPad Prism 5 software. Data were presented as mean \pm standard error of the mean (SEM) of triplicated independent experiments. The in vitro cell experiments were tested by Student's $t$-test or one-way ANOVA test. The chemoresistant assay was tested by two-way ANOVA test. The survival curves were tested by Kaplan-Meier method with log-rank test. The density of bands of Western blot was measured by Image $\mathrm{J}$ software. $P<0.05$ was considered statistically significant.

\section{Results}

\section{PDK4 is identified in chemoresistance ovarian cancer patients and cell lines}

To identify the roles of PDK family members in ovarian cancer, we explored the GSE dataset 51373 containing 12 chemotherapy resistant and 16 chemosensitive patients with high-grade serous epithelial ovarian cancer samples. ${ }^{24} \mathrm{By}$ Affymetrix U133 Plus 2.0 arrays, PDK family transcriptional profile was measured, followed by cluster analysis (Figure 1A). We noticed that the expression of PDK4 was signifi- cantly higher in chemoresistance patients compared to that in sensitive patients (Figure 1B). Given these observations, we further detected the expression of PDK4 in six ovarian cancer cell lines (Figure 1C). Protein level by Western blot indicated that PDK4 was relatively highly expressed in OVISE and OV90 cell lines, while had low expression in ES2 and SKOV3 cell lines (Figure 1D and E).

\section{PDK4 facilitates ovarian cancer cell proliferation and invasion}

To further explore the biological cellular function of PDK4 in ovarian cancer cells, we performed gain-and-loss function analysis in ES2 and OVISE cells, respectively. According to the endogenous expression of PDK4, we performed overexpression of PDK4 in ES2 cells, while knocking down of PDK4 in OVISE cells (Figure 2A). We found that overexpression of PDK4 significantly promoted cell proliferation rate. On the contrary, knockdown of PDK4 by targeting siRNAs decreased OVISE cells growth rate (Figure 2B). Subsequently, we checked the invading
A

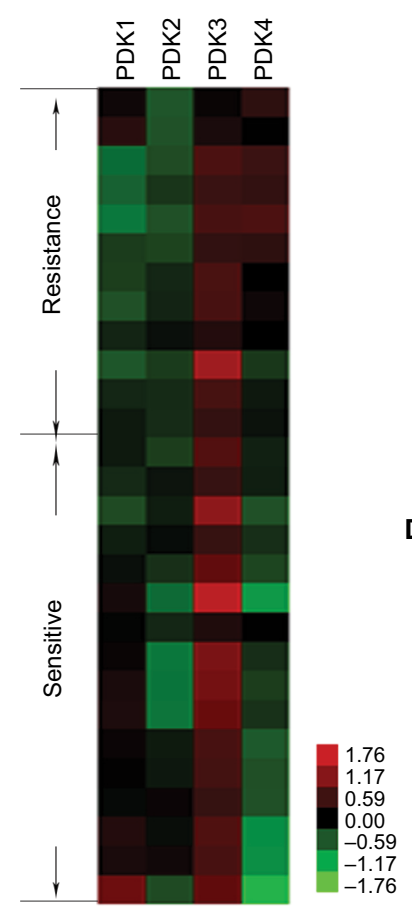

B

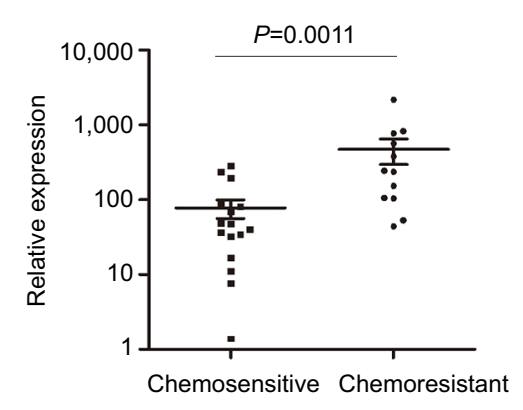

D

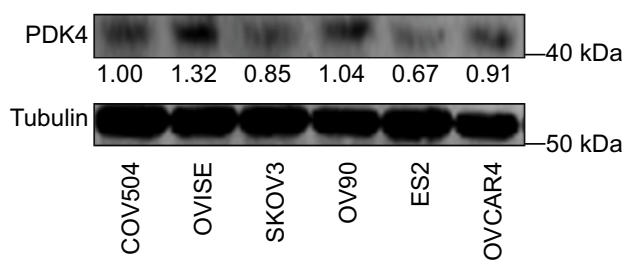

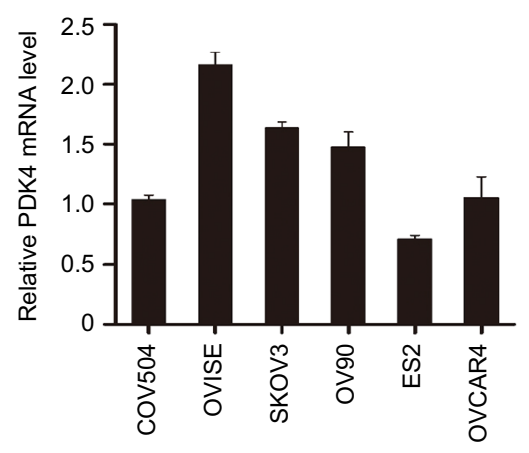

E

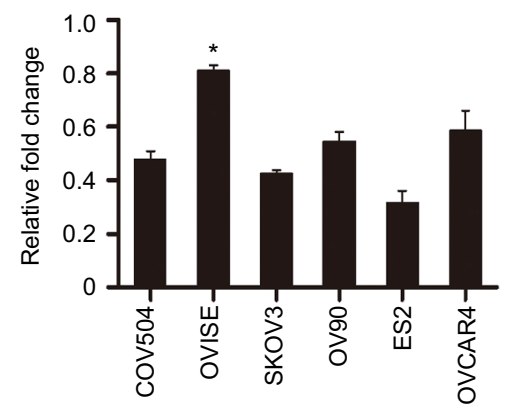

Figure I Identification of PDK4 as a chemoresistance associated gene in ovarian cancer.

Notes: (A) Heat map representing the expression values of pyruvate dehydrogenase kinase family in GSE5I373. Each row indicates 28 cases of ovarian cancer tissue samples. (B) Relative expression of PDK4 in chemosensitive and chemoresistant patients. Statistical significance was analyzed by Mann-Whitney test. (C) mRNA level of PDK4 in six ovarian cancer cell lines was determined by qRT-PCR. The protein level of PDK4 in ovarian cancer cell lines (D) and the quantification of bands by Image $(\mathbf{E})(* P<0.05$, compared to the value in COV504 cell line).

Abbreviation: qRT-PCR, quantitative real-time PCR. 
A

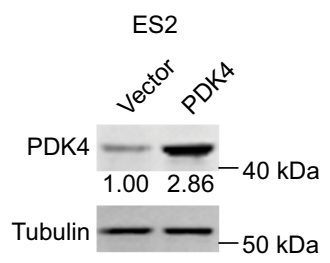

B

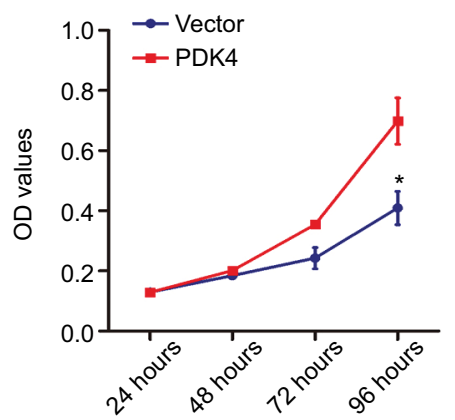

OVISE

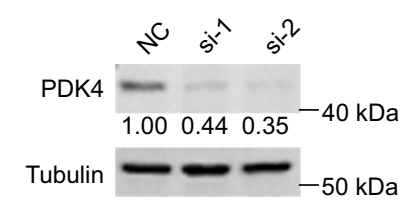

C
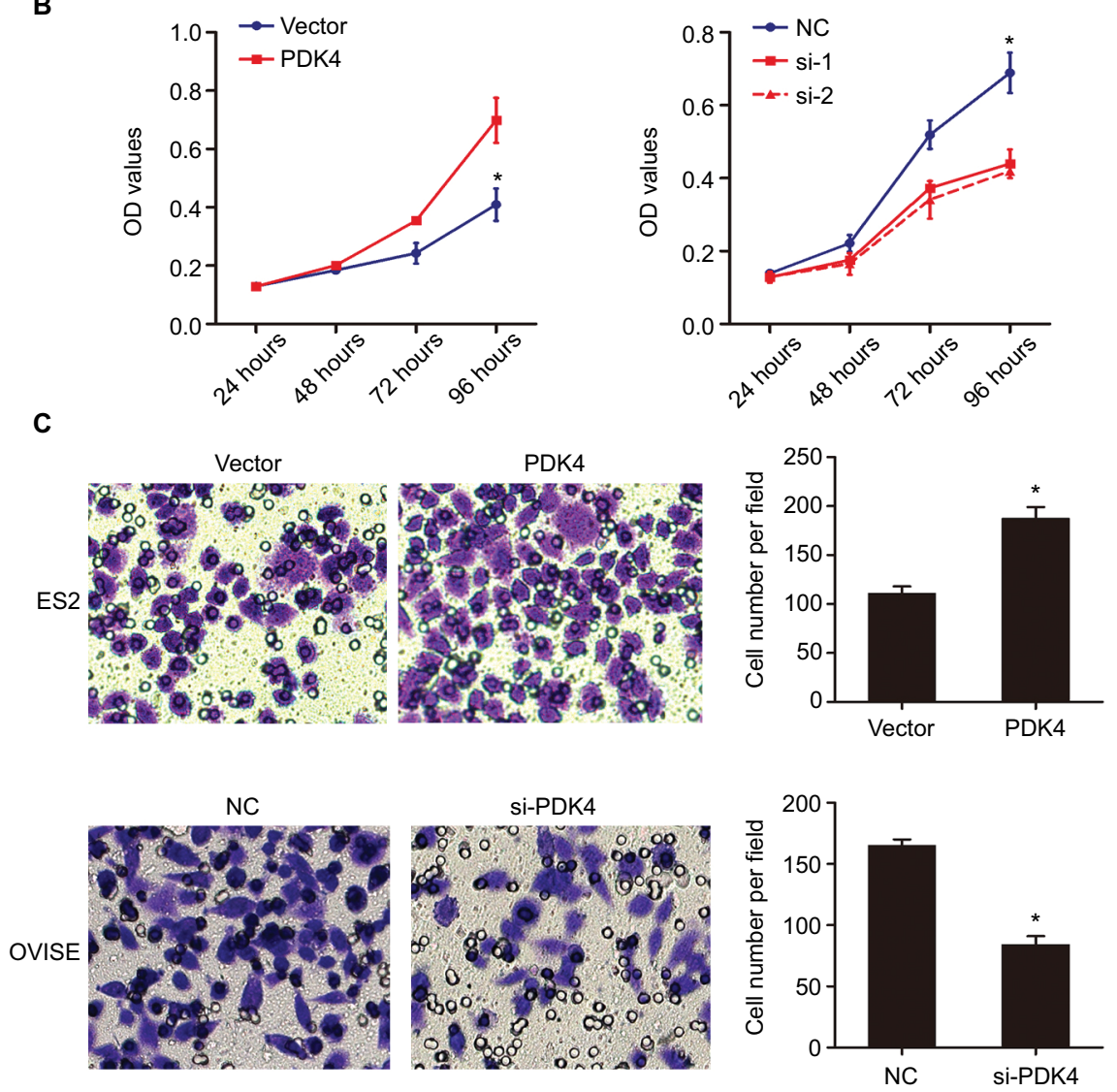

Figure 2 PDK 4 promotes cell proliferation and invasion.

Notes: (A) Expression of PDK4 was determined by Western blot in ES2 transfected with PDK4 plasmid (left panel) or OVISE transfected with two different siRNAs (si-I, si-2, right panel). (B) MTT assay showed that PDK4 overexpression promoted proliferation in ES2 cells (left panel). PDK4 knockdown reduced proliferation in OVISE cells (right panel). The statistical analysis was performed between each group at 96 -hour time point. (C) PDK4 overexpression upregulated invading ability of ES2 cells (upper panel). PDK4 knockdown decreased the invading ability of OVISE cells (lower panel) $(* P<0.05)$. Magnification 200x.

induction ability of PDK4. As shown in Figure 2C, PDK4 overexpression increased ES2 cells invasion, while PDK4 knockdown decreased invading ability. Taken together, these results indicated that PDK4 could induce ovarian cancer cell proliferation and invasion.

\section{PDK4 induces chemoresistance}

Since we found that the expression of PDK4 was higher in chemoresistant patients, we further investigated the effect of PDK4 on chemosensitivity in ovarian cancer cells. We performed cell viability assay using gradient concentration of paclitaxel and cisplatin in PDK4-overexpressed cells or PDK4-knockdown cells (Figure 3A and B). ES2 cells with PDK4 overexpression became much more resistant to paclitaxel (Taxol) and cisplatin (Cis) compared to control cells, while OVISE cells with PDK4 knocking down were more sensitive to two chemodrugs. Detailed data are shown in Table S2. Also, we performed Western blot and immunofluorescence in the four group cells (vector + DMSO, vector + Taxol, PDK4 + DMSO, PDK4 + Taxol), and we found that PDK4 overexpression could decrease the cleaved caspase-3 expression (Figure 3C). Meanwhile, PDK4-knockdown cells sensitized to paclitaxel-triggered apoptosis, and the expression of cleaved caspase- 3 is 
upregulated (Figure 3D). Likewise, the apoptosis assay by flow cytometry also confirmed that PDK4 conferred chemoresistance in ovarian cancer cells. We noticed that the Taxol-induced apoptosis was attenuated after PDK4 overexpression, while significantly elevated after PDK4 knocking down (Figure 3E and F).

\section{PDK4 increases stemness properties and CDI33+ subpopulations in ovarian cancer cells}

To investigate the roles of PDK4-induced chemoresistance, we performed spheroid formation assay to evaluate the effect of PDK4 in cell self-renewal ability. PDK4 overexpression promoted spheroid formation as we seeded cells in ultra-low attachment plates (Figure 4A). However, knockdown of PDK4 reduced spheroid volume and numbers. Moreover, the CD133+ percentage of cells with PDK4 overexpression was significantly greater than that of the control cells, while knockdown of PDK4 inhibited CD133+ subpopulation cells (Figure 4B). Notably, we observed that the expression of several stem cell-associated genes, such as Bmil, Nanog and Oct4, was increased significantly after PDK4 overexpression. On the contrary, knockdown of PDK4 reduced these genes expression (Figures $4 \mathrm{C}$ and $\mathrm{S} 1$ ).

\section{PDK4 promotes tumorigenesis in vivo}

To further confirm the oncogenic role of PDK4 in ovarian cancer, a total of $1 \times 10^{6}$ ES2 cells overexpressing PDK4 and control cells were subcutaneously injected into the nude mice, respectively. After 4 weeks, we found that overexpression of PDK4 significantly promoted tumor growth compared to control group (Figure $5 \mathrm{~A}$ and $\mathrm{B}$ ). Also, the tumor weight was significantly elevated in group that received PDK4-overexpressing cells (Figure 5C). Moreover, immunohistochemical staining of Ki67, a proliferation marker, demonstrated the growth promotion effect of PDK4 (Figure 5D).

\section{PDK4 is upregulated in ovarian cancer tissues and correlated with poor survival}

To delineate the expression and function of PDK4 in human ovarian cancer tissues, we employed tissue microarrays containing 75 cases of ovarian cancer tissues and 5 cases of adjacent non-cancer tissues for IHC staining. Predominant cytoplasmic staining was observed in ovarian cancer cells, and the expression of PDK4 in ovarian cancer tissues was significantly higher than that in benign tissues (Figure 6A). We then searched the mRNA expression level of PDK4 in GSE26712 containing 185 primary ovarian tumors and 10 normal ovarian surface epithelium. The results showed that PDK4 expression was significantly upregulated in ovarian cancer tissues compared with normal tissues (Figure 6B). Furthermore, we detected the prognostic value of PDK4 in ovarian cancer using Kaplan-Meier plotter. As shown in Figure 6C and $\mathrm{D}$, both the overall survival and progression-free survival were shorter in ovarian cancer patients with higher PDK4 expression, indicating that PDK4 could be potential prognostic factor of ovarian cancer.

\section{Discussion}

Ovarian cancer is one of the most aggressive gynecological malignancies worldwide. Chemoresistance and metastasis are the major factors that are responsible for the unfavorable outcomes. The present study focused on PDK4, member of the PDK family, which was identified as the chemoresistance-associated genes based on the public GEO database. By ectopic overexpression and silencing of PDK4, we demonstrated that PDK4 promoted cell proliferation, invasion and conferred ovarian cancer cells resistant to chemodrug-induced apoptosis through inducing cell stemness properties. Also, PDK4 promoted tumorigenesis in vivo. In tissue level, PDK4 is dominantly expressed in ovarian cancer tissues compared to benign tissues and significantly correlated with poor prognosis. Our finding indicated that PDK4-induced chemoresistance played an important role in ovarian cancer.

PDK4 has been implicated in several cancers. It is reported that PDK4 could be upregulated in endometrial cancer cells PRB23 treated with progesterone receptor agonist R5020, indicating that during hormone treatment, endometrial cancer cells preferred to prevent the conversion of pyruvate to acetyl-CoA. ${ }^{25}$ It was reported that PDK knockdown inhibited proliferation in lung cancer cells while Brugge et al observed that overexpression of PDK4 decreased PDH flux, de novo lipogenesis and cell proliferation. ${ }^{26,27}$ These might because that PDK4 decrease acetyl-CoA, which was the precursor for fatty acid synthesis and energy production. However, tumor cells undergo unique metabolism reprogramming which is identified as Warburg effect and the fully production of acetyl-CoA was not much needed. ${ }^{28}$ Moreover, PDK4 could be regulated by several critical transcriptional activator and miRNAs, such as PGC-1, FOXO1, Rb-E2F1 and miR-182. ${ }^{29-32}$ In $\mathrm{C} 2 \mathrm{C} 12$ cells, PDK4 was induced by LPS via JNK pathway, 
A

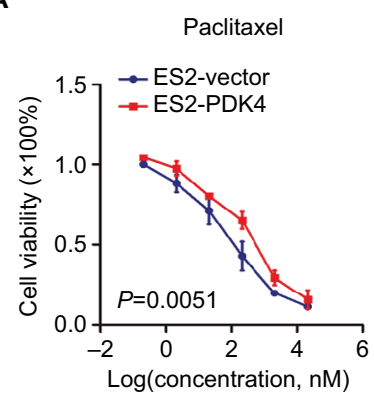

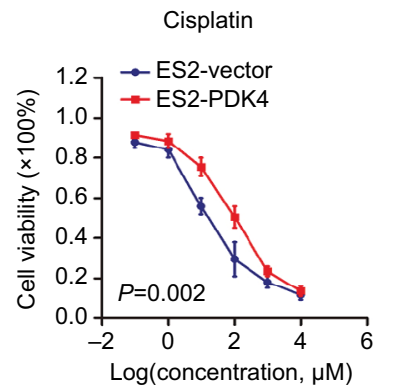

B
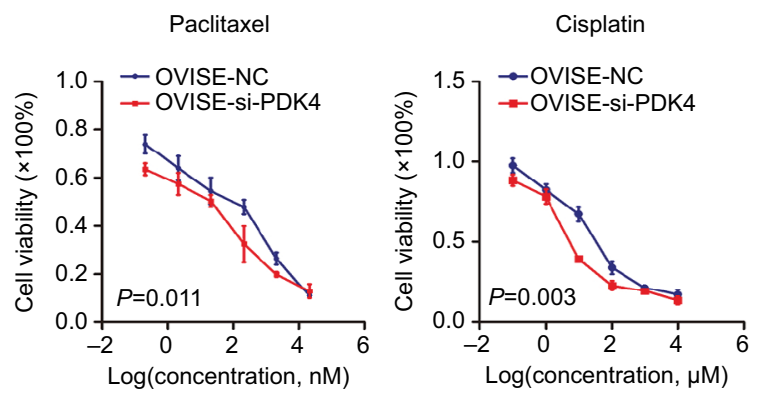

C
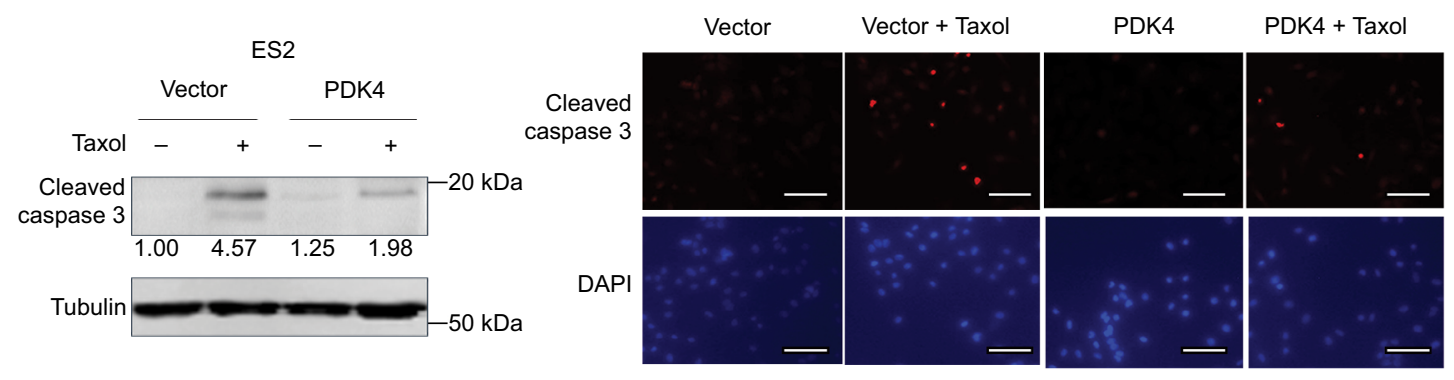

D
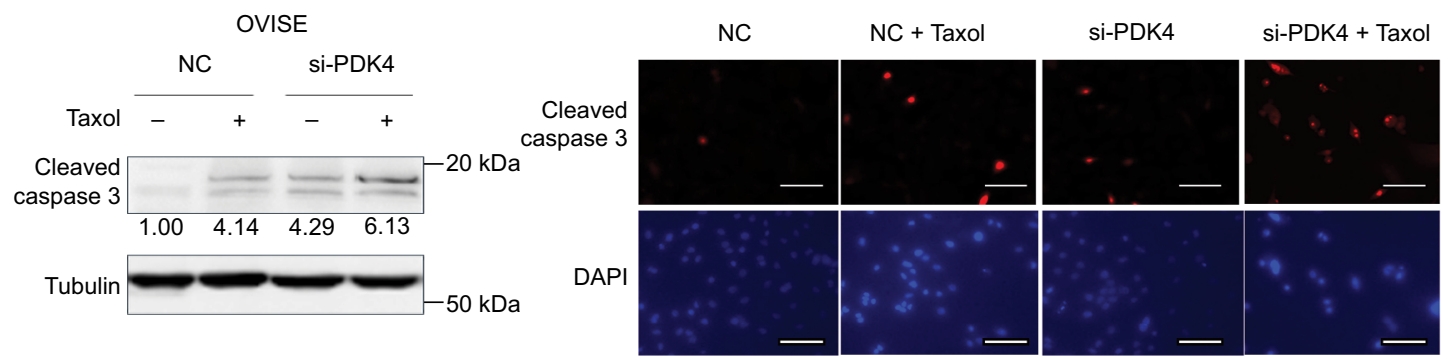

E

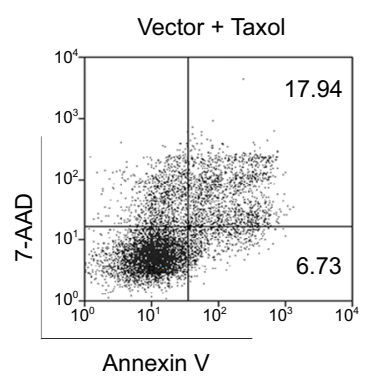

ES2

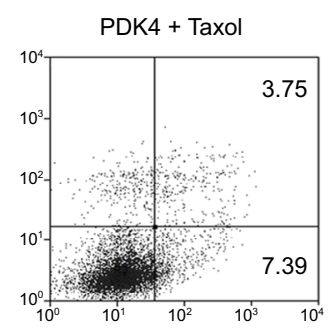

$\mathbf{F}$
OVISE

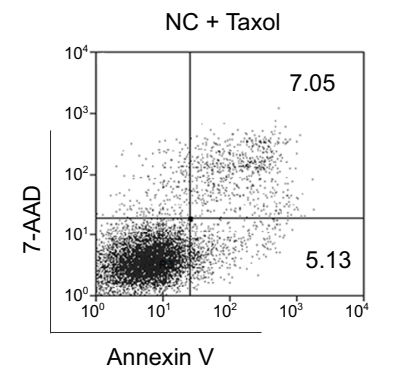

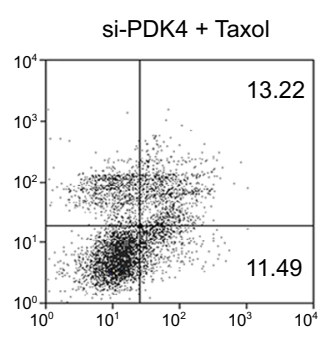
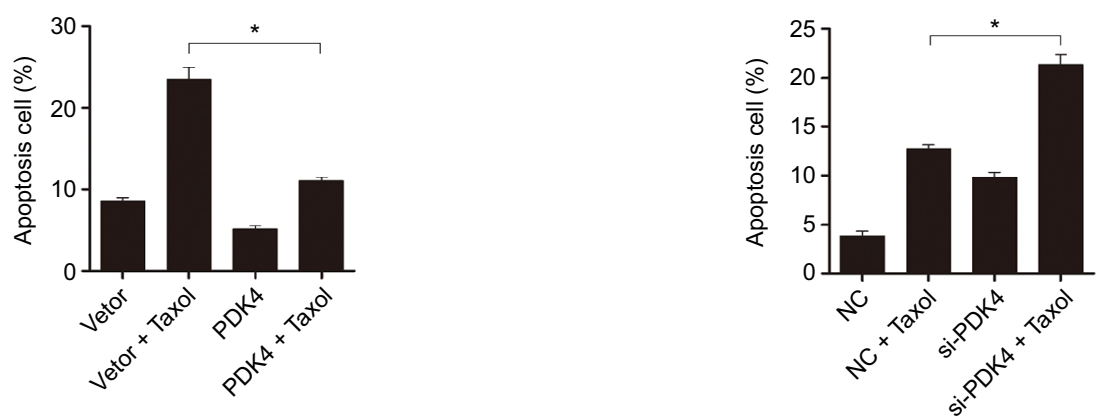

Figure 3 Overexpression of PDK4 confers ovarian cancer cells resistant to paclitaxel and cisplatin.

Notes: Cell viability assay was performed in PDK4-overexpressed ES2 cells (A) and PDK4-knocdown OVISE cells (B) treated with different concentration of paclitaxel (Taxol) and cisplatin (Cis). (C) Overexpression of PDK4 decreased expression of cleaved caspase-3 which induced by Taxol as evaluated by Western blot and immunofluorescence. (D) Knocking down of PDK4 increased the expression of cleaved caspase-3 (bar, $100 \mu \mathrm{m}$ ). Paclitaxel-induced cell apoptosis was determined in ES2 cells transfected with PDK4 (E) and OVISE cells transfected with siRNAs $(\mathbf{F})(* P<0.05)$. 
A

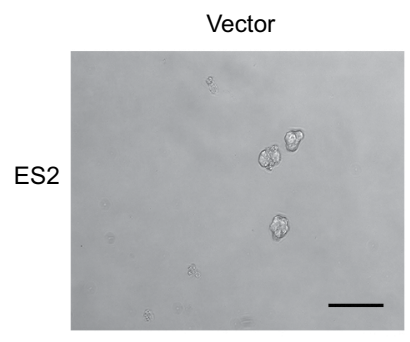

NC

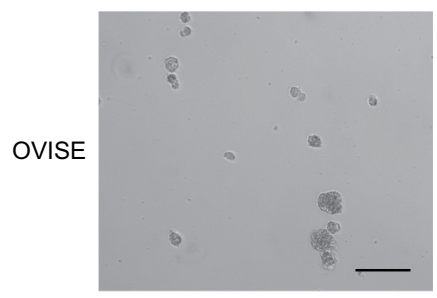

PDK4

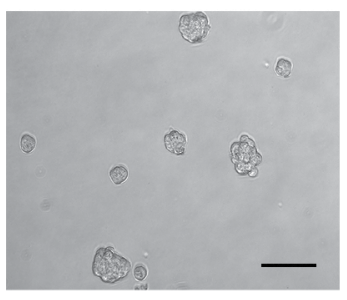

si-PDK4

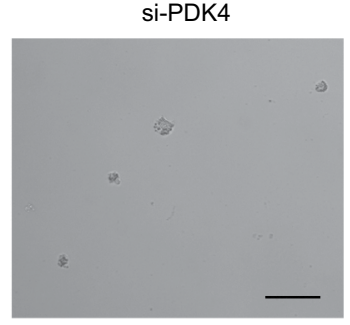

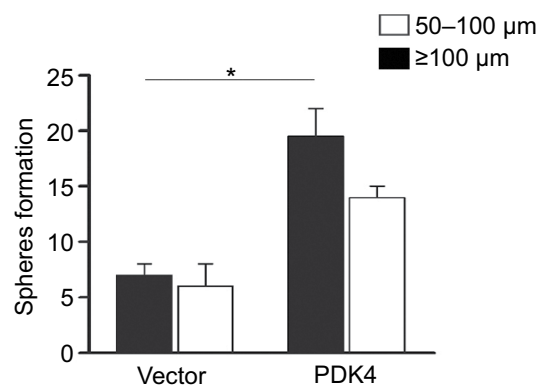

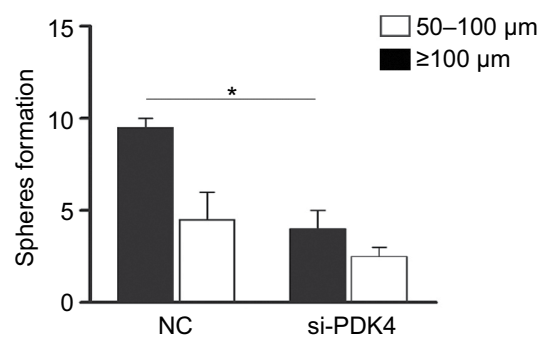

B
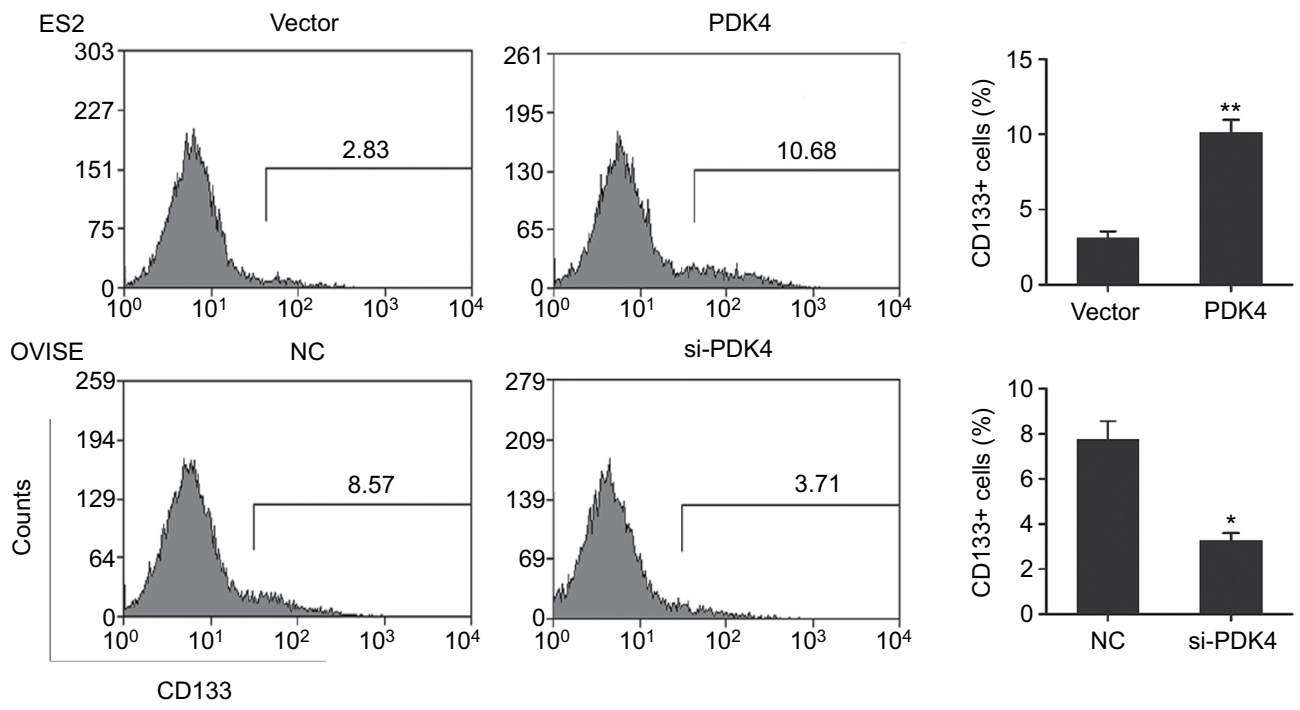

C
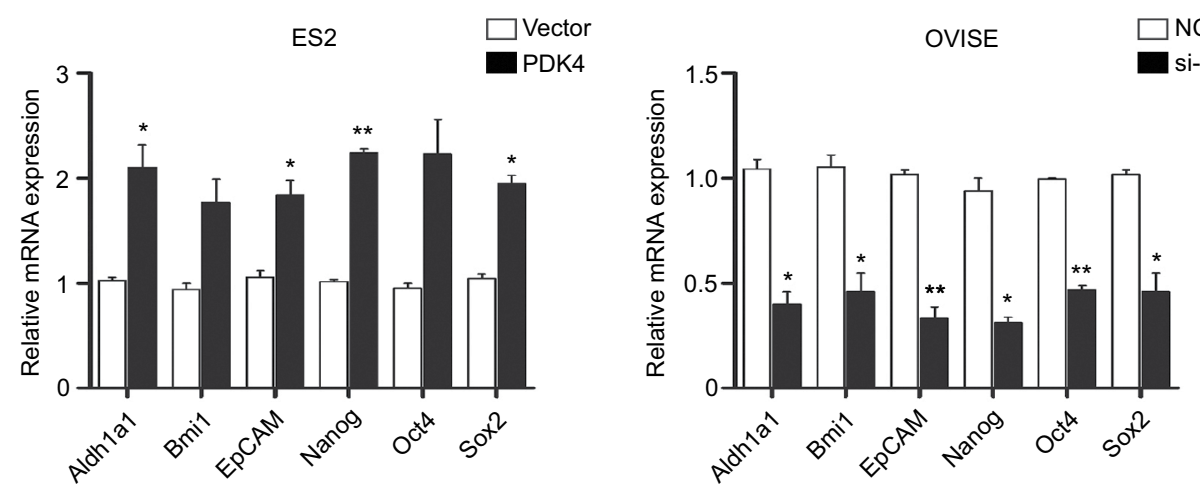

Figure 4 PDK4 enhances stemness and enriches CDI33+ subpopulations of ovarian cancer cells.

Notes: (A). Spheroid formation assay for ES2 cells transfected with PDK4 and OVISE cells transfected with siRNA. Representative images (left panel) and statistical measurement (right panel). (B) CDI33+ subpopulations of aforementioned cells. Representative FACS profiles. (C) Stem cell-associated gene expression was measured by qRT-PCR in aforementioned cells $(* P<0.05$, $* * P<0.01)$.

Abbreviations: FACS, fluorescence-activated cell sorting; qRT-PCR, quantitative real-time PCR. 


\section{A}

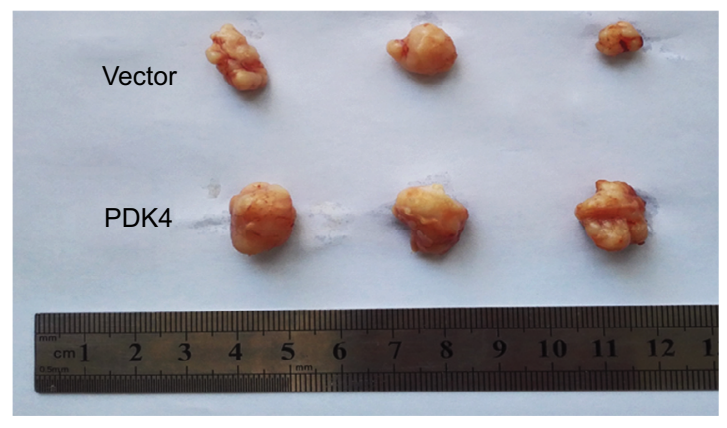

C

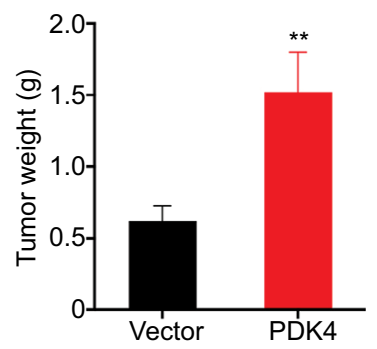

D
B

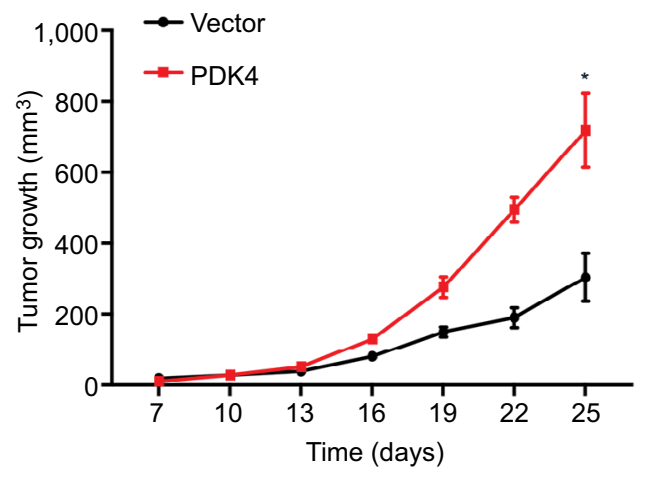

Figure 5 PDK4 promotes tumor growth in nude mice.

Notes: (A) Representative images of tumors derived from PDK4-overexpressing cells and control. (B) Tumors from PDK4-overexpressing cells grew faster than control group. (C) The tumor weight of two groups. (D) The IHC of Ki67 was performed in tumor sections of each group (left), and the statistical results were indicated (right) $(* P<0.05, * * P<0.01)$. Magnification I00x. Scale bar $100 \mu \mathrm{m}$.

Abbreviation: IHC, immunohistochemical.
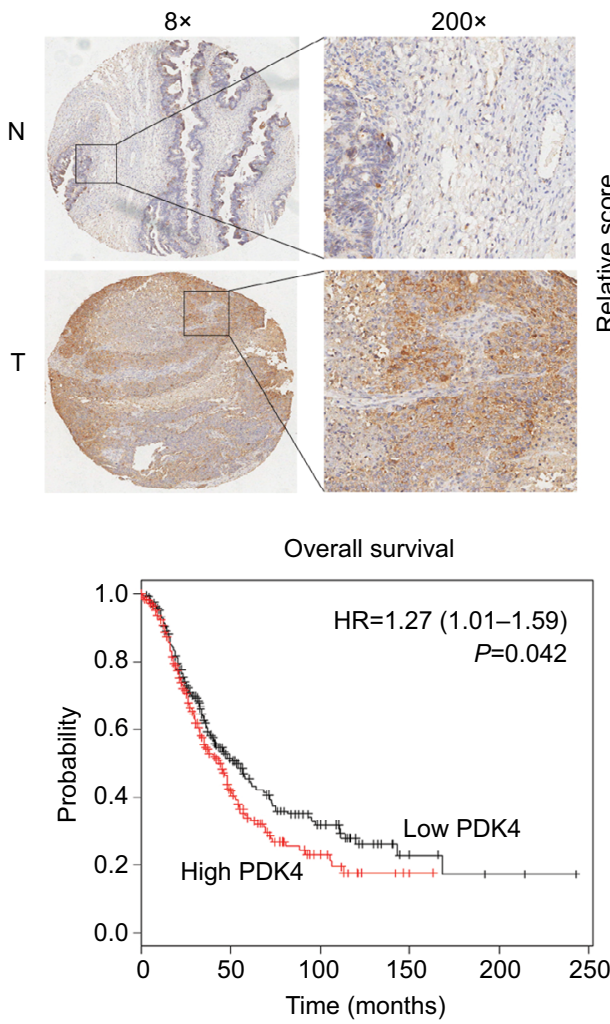
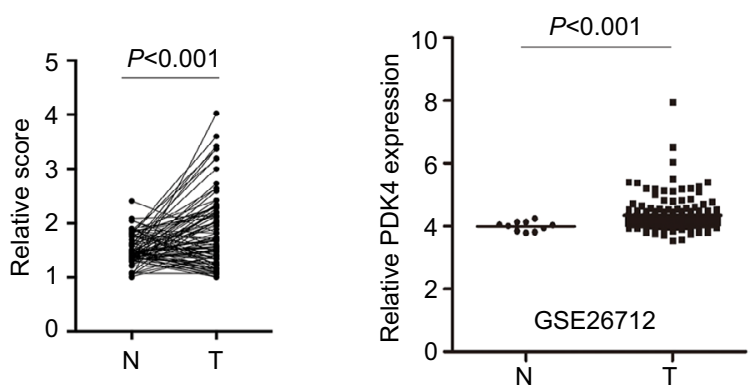

D

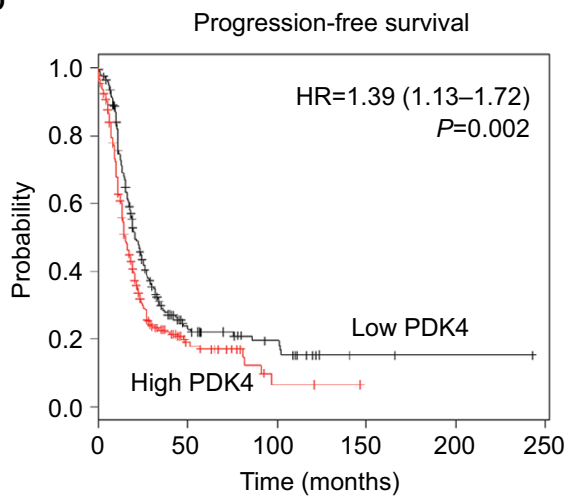

Figure 6 PDK4 expression is elevated in ovarian cancer tissues and associated with poor prognosis.

Notes: (A) Representative images of PDK4 staining in benign tissues and tumor tissues (left). Scoring for the IHC was indicated (right). (B) Relative expression of PDK4 was upregulated in tumor tissues (T) compared with that in normal tissues (N) using GSE267I2 dataset. Overall survival analysis (C) and progression free-survival analysis (D) of ovarian cancer patients with PDK4 (probe: 225207_at) expression by Kaplan-Meier plotter database.

Abbreviation: IHC, immunohistochemical. 
suggesting the new mechanism that LPS altered glucose metabolism. ${ }^{33}$ So PDK4 plays the balancer between glycolysis and oxidative phosphorylation, while the specific mechanism of that PDK4 in cancer remains to be further elucidated.

To address the biological functions and prognostic significance of PDK4 in ovarian cancer, we performed in vitro cell experiments and IHC on tumor tissues. The results revealed that $\mathrm{PDK} 4$ overexpression significantly promoted ovarian cancer cell self-renewal ability which was evidenced by spheroid formation assay and FACS. These observations were compatible with previous study in hepatocellular carcinoma that inhibition of PDK4 and LDHA suppressed stemness characteristics and overcome resistance to sorafenib. ${ }^{13}$ Recently, FAB210B was reported to regulate PDK4 and affect Warburg effect, thereby, modulating ovarian cancer metastasis. ${ }^{34}$ Moreover, PDK4 was reported to be upregulated in high grade bladder cancer and associated with the cisplatin resistance of bladder cancer cells. ${ }^{35}$ The tumor stem cells were well recognized by the self-renewal and chemoresistance abilities, so PDK4induced enhancement of self-renewal contributed to the ovarian cancer cells' malignancy. ${ }^{36-38}$ We also employed Kaplan-Meier plotter database to test PDK4 in a large scale and found that elevated expression of PDK4 was significantly correlated with both overall survival and progression-free survival of ovarian cancer patients.

In summary, we showed that PDK4 was overexpressed in ovarian cancer tissues and correlated with increased chemoresistance and poor prognosis. PDK4 promoted ovarian cancer cell proliferation, invasion and resistant to chemotherapy-induced apoptosis. Moreover, PDK4 induced cell stemness properties and stem-associated genes expression. These findings could provide new insight into the pathogenesis of ovarian cancer development and chemoresistance that PDK4 serves as a potential target for treatments.

\section{Disclosure}

The authors report no conflicts of interest in this work.

\section{References}

1. Torre LA, Bray F, Siegel RL, Ferlay J, Lortet-Tieulent J, Jemal A. Global cancer statistics, 2012. CA Cancer J Clin. 20152015;65(2):87-108.

2. Siegel RL, Miller KD, Jemal A, Statistics C. Cancer statistics, 2015. CA Cancer J Clin. 2015;65(1):5-29.

3. Song YS, Kim HS, Aoki D, Dhanasekaran DN, Tsang BK. Ovarian cancer. Biomed Res Int. 2014;2014:764323.

4. Darcy KM, Birrer MJ. Translational research in the Gynecologic Oncology Group: evaluation of ovarian cancer markers, profiles, and novel therapies. Gynecol Oncol. 2010;117(3):429-439.
5. Jayson GC, Kohn EC, Kitchener HC, Ledermann JA. Ovarian cancer. Lancet. 2014;384(9951):1376-1388.

6. van der Heiden MG, Cantley LC, Thompson CB. Understanding the Warburg effect: the metabolic requirements of cell proliferation. Science. 2009;324(5930):1029-1033.

7. Kato M, Wynn RM, Chuang JL, et al. Structural basis for inactivation of the human pyruvate dehydrogenase complex by phosphorylation: role of disordered phosphorylation loops. Structure. 2008;16(12):1849-1859.

8. Jeoung NH. Pyruvate dehydrogenase kinases: therapeutic targets for diabetes and cancers. Diabetes Metab J. 2015;39(3):188-197.

9. Leclerc D, Pham DN, Lévesque N, et al. Oncogenic role of PDK4 in human colon cancer cells. Br J Cancer. 2017;116(7):930-936.

10. Li G, Li M, Hu J, et al. The microRNA-182-PDK4 axis regulates lung tumorigenesis by modulating pyruvate dehydrogenase and lipogenesis. Oncogene. 2017;36(7):989-998.

11. Woolbright BL, Choudhary D, Mikhalyuk A, et al. The role of pyruvate dehydrogenase kinase-4 (PDK4) in bladder cancer and chemoresistance. Mol Cancer Ther. 2018;17(9):2004-2012.

12. Srivastava N, Kollipara RK, Singh DK, et al. Inhibition of cancer cell proliferation by PPAR $\gamma$ is mediated by a metabolic switch that increases reactive oxygen species levels. Cell Metab. 2014;20(4): 650-661.

13. Song $\mathrm{K}$, Kwon $\mathrm{H}$, Han $\mathrm{C}$, et al. Active glycolytic metabolism in CD133(+) hepatocellular cancer stem cells: regulation by MIR-122. Oncotarget. 2015;6(38):40822-40835.

14. Dlamini Z, Ntlabati P, Mbita Z, Shoba-Zikhali L. Pyruvate dehydrogenase kinase 4 (PDK4) could be involved in a regulatory role in apoptosis and a link between apoptosis and insulin resistance. Exp Mol Pathol. 2015;98(3):574-584.

15. Liu Z, Chen X, Wang Y, et al. PDK4 protein promotes tumorigenesis through activation of cAMP-response element-binding protein (CREB)Ras homolog enriched in brain (RHEB)-mTORC1 signaling cascade. J Biol Chem. 2014;289(43):29739-29749.

16. Wang B, Ma A, Zhang L, et al. POH1 deubiquitylates and stabilizes E2F1 to promote tumour formation. Nat Commun. 2015;6:8704.

17. Qian Y, Wang B, Ma A, et al. USP16 downregulation by carboxyl-terminal truncated HBx promotes the growth of hepatocellular carcinoma cells. Sci Rep. 2016;6:33039.

18. Yu Y, Gaillard S, Phillip JM, et al. Inhibition of spleen tyrosine kinase potentiates paclitaxel-induced cytotoxicity in ovarian cancer cells by stabilizing microtubules. Cancer Cell. 2015;28(1):82-96.

19. Samuel P, Pink RC, Caley DP, Currie JM, Brooks SA, Carter DR. Over-expression of miR-31 or loss of KCNMA1 leads to increased cisplatin resistance in ovarian cancer cells. Tumour Biol. 2016;37(2):2565-2573.

20. Yang Z, Zhang L, Ma A, et al. Transient mTOR inhibition facilitates continuous growth of liver tumors by modulating the maintenance of CD133+ cell populations. PLoS One. 2011;6(12):e28405.

21. de Hoon MJ, Imoto S, Nolan J, Miyano S. Open source clustering software. Bioinformatics. 2004;20(9):1453-1454.

22. Saldanha AJ. Java Treeview - extensible visualization of microarray data. Bioinformatics. 2004;20(17):3246-3248.

23. Gyorffy B, Lánczky A, Szállási Z. Implementing an online tool for genome-wide validation of survival-associated biomarkers in ovariancancer using microarray data from 1287 patients. Endocr Relat Cancer. 2012;19(2):197-208.

24. Koti M, Gooding RJ, Nuin P, et al. Identification of the IGF1/PI3K/ $\mathrm{NF}$ B/ERK gene signalling networks associated with chemotherapy resistance and treatment response in high-grade serous epithelial ovarian cancer. BMC Cancer. 2013;13:549.

25. Pant A, Lee II, Lu Z, Rueda BR, Schink J, Kim JJ. Inhibition of AKT with the orally active allosteric AKT inhibitor, MK-2206, sensitizes endometrial cancer cells to progestin. PLoS One. 2012;7(7):e41593.

26. Bonnet S, Archer SL, Allalunis-Turner J, et al. A mitochondria$\mathrm{K}+$ channel axis is suppressed in cancer and its normalization promotes apoptosis and inhibits cancer growth. Cancer Cell. 2007;11(1):37-51. 
27. Grassian AR, Metallo CM, Coloff JL, Stephanopoulos G, Brugge JS Erk regulation of pyruvate dehydrogenase flux through PDK4 modulates cell proliferation. Genes Dev. 2011;25(16):1716-1733.

28. Yang W, Zheng Y, Xia Y, et al. ERK1/2-dependent phosphorylation and nuclear translocation of PKM2 promotes the Warburg effect. Nat Cell Biol. 2012;14(12):1295-1304.

29. Wende AR, Huss JM, Schaeffer PJ, Giguère V, Kelly DP. PGC-1alpha coactivates PDK4 gene expression via the orphan nuclear receptor ERRalpha: a mechanism for transcriptional control of muscle glucose metabolism. Mol Cell Biol. 2005;25(24):10684-10694.

30. Furuyama T, Kitayama K, Yamashita H, Mori N. Forkhead transcription factor FOXO1 (FKHR)-dependent induction of PDK4 gene expression in skeletal muscle during energy deprivation. Biochem J. 2003;375(Pt 2):365-371.

31. Hsieh MC, das D, Sambandam N, Zhang MQ, Nahlé Z. Regulation of the PDK4 isozyme by the Rb-E2F1 complex. J Biol Chem. 2008;283(41):27410-27417.

32. Li G, Li M, Hu J, et al. The microRNA-182-PDK4 axis regulates lung tumorigenesis by modulating pyruvate dehydrogenase and lipogenesis. Oncogene. 2017;36(7):989-998.
33. Park H, Jeoung NH. Inflammation increases pyruvate dehydrogenase kinase 4 (PDK4) expression via the Jun N-terminal kinase (JNK) pathway in C2C12 cells. Biochem Biophys Res Commun. 2016;469(4): 1049-1054.

34. Sun S, Liu J, Zhao M, et al. Loss of the novel mitochondrial protein FAM210B promotes metastasis via PDK4-dependent metabolic reprogramming. Cell Death Dis. 2017;8(6):e2870.

35. Woolbright BL, Choudhary D, Mikhalyuk A, et al. The role of pyruvate dehydrogenase kinase-4 (PDK4) in bladder cancer and chemoresistance. Mol Cancer Ther. 2018;17(9):2004-2012.

36. Liu G, Yuan X, Zeng Z, et al. Analysis of gene expression and chemoresistance of CD133+ cancer stem cells in glioblastoma. Mol Cancer. 2006;5:67.

37. Alvero AB, Chen R, Fu HH, et al. Molecular phenotyping of human ovarian cancer stem cells unravels the mechanisms for repair and chemoresistance. Cell Cycle. 2009;8(1):158-166.

38. Hu L, McArthur C, Jaffe RB. Ovarian cancer stem-like sidepopulation cells are tumourigenic and chemoresistant. Br J Cancer. 2010;102(8):1276-1283. 


\section{Supplementary materials}

Table SI Primer sequences

\begin{tabular}{|c|c|c|c|}
\hline Genbank accession & Gene name & Primer sequence $\left(5^{\prime}-3^{\prime}\right)$ & Amplicon length (bp) \\
\hline \multirow[t]{2}{*}{ NM_00I256799 } & \multirow[t]{2}{*}{ GAPDH } & CTGGGCTACACTGAGCACC & \multirow[t]{2}{*}{101} \\
\hline & & AAGTGGTCGTTGAGGGCAATG & \\
\hline \multirow[t]{2}{*}{ NM_002612 } & \multirow[t]{2}{*}{ PDK4 } & GGAGCATTTCTCGCGCTACA & \multirow[t]{2}{*}{117} \\
\hline & & ACAGGCAATTCTTGTCGCAAA & \\
\hline \multirow[t]{2}{*}{ NM_000689 } & \multirow[t]{2}{*}{ Aldhlal } & GCACGCCAGACTTACCTGTC & \multirow[t]{2}{*}{129} \\
\hline & & CСTCCTCAGTTGCAGGATTAAAG & \\
\hline \multirow[t]{2}{*}{ NM_005I80 } & \multirow[t]{2}{*}{ Bmil } & TGAAGATAGAGGAGAGGTTGC & \multirow[t]{2}{*}{172} \\
\hline & & CTGCTGGGCATCGTAAGTAT & \\
\hline \multirow[t]{2}{*}{ NM_002354 } & \multirow[t]{2}{*}{ ЕрСАM } & CTGGCCGTAAACTGCTTTGT & \multirow[t]{2}{*}{181} \\
\hline & & AGCCCATCATTGTTCTGGAG & \\
\hline \multirow[t]{2}{*}{ NM_024865 } & \multirow[t]{2}{*}{ Nanog } & GTCCCGGTCAAGAAACAGAA & \multirow[t]{2}{*}{229} \\
\hline & & TGCGTCACACCATTGCTATT & \\
\hline \multirow[t]{2}{*}{ NM_00270I } & \multirow[t]{2}{*}{ Oct4 } & ATTCAGCCAAACGACCATCT & \multirow[t]{2}{*}{232} \\
\hline & & ACACTCGGACCACATCCTTC & \\
\hline \multirow[t]{2}{*}{ NM_003I06 } & \multirow[t]{2}{*}{ Sox2 } & ATGGGTTCGGTGGTCAAGT & \multirow[t]{2}{*}{295} \\
\hline & & ATGTGTGAGAGGGGCAGTGT & \\
\hline
\end{tabular}

Table S2 The IC50 value of each group

\begin{tabular}{|l|l|l|}
\hline & IC50 of paclitaxel $(\mathrm{nM})$ & IC50 of cisplatin $(\mu \mathrm{M})$ \\
\hline ES2-vector & $20.19 \pm 7.97$ & $16.68 \pm 4.39$ \\
\hline ES2-PDK4 & $49.41 \pm 4.36$ & $101.24 \pm 3.57$ \\
\hline OVISE-NC & $76.47 \pm 8.45$ & $35.17 \pm 2.97$ \\
\hline OVISE-si-PDK4 & $15.92 \pm 3.78$ & $6.54 \pm I .85$ \\
\hline
\end{tabular}

ES2

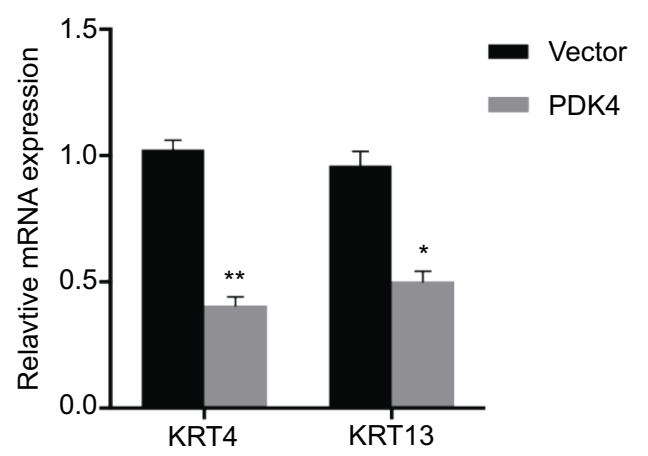

OVISE

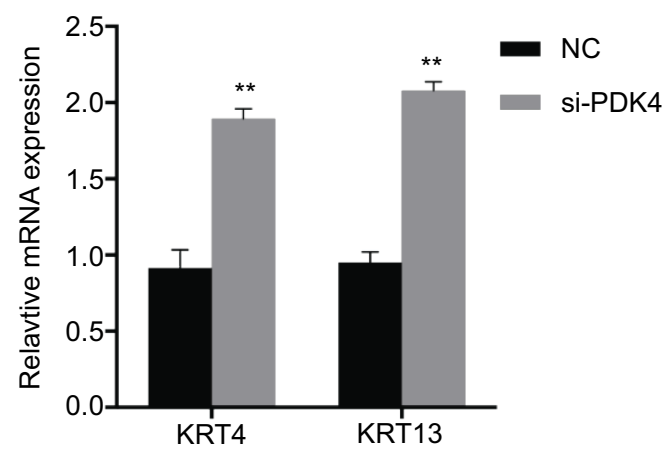

Figure SI The mRNA expressions of differentiation marker KRT4 and KRTI3 were examined after PDK4 overexpression or knocking down by $R T-P C R(* P<0.05$, **P $<0.01)$.

Cancer Management and Research

\section{Publish your work in this journal}

Cancer Management and Research is an international, peer-reviewed open access journal focusing on cancer research and the optimal use of preventative and integrated treatment interventions to achieve improved outcomes, enhanced survival and quality of life for the cancer patient. The manuscript management system is completely online and includes a very quick and fair peer-review system, which is all easy to use. Visit http://www.dovepress.com/testimonials.php to read real quotes from published authors. 